\title{
Publishing Undergraduate Engineering Designs: A Case Study
}

\author{
Medhat Moussa \\ Associate Professor \\ School of Engineering \\ University of Guelph \\ mmoussa@uoguelph.ca
}

\author{
William David Lubitz \\ Assistant Professor \\ School of Engineering \\ University of Guelph \\ wlubitz@uoguelph.ca
}

\author{
Antony Savich \\ Doctoral Candidate \\ School of Engineering \\ University of Guelph \\ asavich@uoguelph.ca
}

\begin{abstract}
Many engineering schools require senior undergraduate students to complete term-long design projects. These projects are normally communicated using a major design report that is submitted for instructor evaluation, with no expectation of any additional audience for the work. This paper presents a case study in publishing undergraduate engineering designs using a short conference style paper format. The short paper has been integrated into a projectbased third year design course at the School of Engineering, University of Guelph. The course is taken by all engineering students at the same time and the design projects vary from environmental, to bioprocess and software design projects. The procedure used to help students formulate their designs as short papers is outlined. The papers are published in course proceedings that are publicly available through the University of Guelph Library's Atrium web portal. To ensure uniform style and format, papers are prepared in LaTeX, which is also used to assemble the final proceedings. A LaTeX kit will be made available for other universities that wish to follow the same procedure.
\end{abstract}

\section{Introduction}

"Final reports" are common to many engineering courses and almost all courses involving group design projects. These reports typically document a complicated design process, from problem definition through to a final design, and are almost always submitted at the end of the semester.

The readers of final reports are usually course instructors, and students write the reports for this audience. In practice, the primary purpose of a report submitted at the end of a course is summative evaluation of student performance in order to assign course marks. The result is that while students invest significant time writing these reports, and instructors invest more time reading and evaluating them, there is little real performance feedback to the students. The report is a terminal document. In the authors' experience, many students do not collect end-of-term reports, and so by definition never receive feedback, and likely do not reflect, on their report writing skills as embodied in the end-of-term report.

In 2007 the authors sought an additional end-ofterm writing exercise that would have educational value for students while also expanding the range of student engineering writing experiences. Addressing some of the shortcomings of the final report, such as the inherent lack of feedback and learning, and the limited audience for the report, was a priority. The preparation of short (two page), conference-style papers was initiated.

The papers are kept to a two page limit to encourage concise writing. Unlike in a large report, the students have to go through the exercise of determining the most important information to communicate, and how to communicate that information clearly and concisely. Students are instructed to write the short papers for a broad audience with no prior knowledge of the project. Since the paper is short and is based on the material in the final report, the preparation of the paper does not take most students. The short format also allowed the use of a reviewing process as will be outlined later.

\section{Case study context}

The authors teach a third year project-based design course in the School of Engineering at the University of Guelph. Engineering and Design III (ENGG*3100) is the third course in a four course sequence focussed on teaching and practicing engineering design. These courses are taken by all engineering students at the University of Guelph. The first two courses expose students to fundamentals of design, and include group design projects to allow students to practice and reinforce their design skills.

ENGG*3100 is centered on the completion of a semester-long open-ended design project by groups of 
three to four students. All of the projects used in the course are based on an identified need of an outside collaborating industry or organization. Almost all course activities, including lectures, are centered around the project. The primary learning objectives are to continue to reinforce design skills taught in the prior courses, develop skills in research, integration and application of knowledge, and learn and reinforce engineering communication, with an emphasis on written documents. Several written deliverables are required during the course. Student groups were, and still are, required to submit a large final report that documents both the semester long design process and their final design solution.

\section{Submission and evaluation process}

The short paper has been included as an element of ENGG*3100 for three years. The implementation has been similar each year, and major changes are not currently planned for the next course offering.

\subsection{Integration in the course}

Students are informed in the course outline, and also several times during initial lectures, that they will be writing a short paper on their design project, and the short paper will be publicly available on the internet after the conclusion of the course. In three years, there have been no issues raised by the students about this, and no requests to not publish a paper on the web portal. (Any requests to not publish a paper would be honoured.)

Student groups are told they must write a short "conference paper" that summarizes their project, and that it is expected this short paper will be "distilled from the final report." Since most students have little prior experience writing short papers, the expectations for short papers are reviewed in lecture before the draft papers are due. Additionally, the short paper proceedings from prior course offerings are available to the students (and the rest of the world) to give them a sense of expectations.

The short paper assignment is a two part process: student groups first submit a draft paper that is reviewed ungraded, and after receiving feedback and making revisions submit an improved final paper. The timing of this process is an important part of course planning.

Draft papers must be submitted approximately one week before the final paper is due. In a 12 week semester, the draft is due in the middle of week 11 , or on the Monday of week 12. Students are instructed to submit a PDF version of their draft paper through the course web site (currently Desire to Learn, formerly
WebCT), to facilitate individual feedback. After the submission deadline, all the draft papers are immediately distributed to all instructors (faculty and teaching assistants) who critically review the papers. Comments are given on the papers that identify writing or content issues and suggest improvements. The reviewed papers are returned to the students within 48 hours.

The rapid reviewing, which is only possible due to the two page size limit, allows the students most of a week to revise their paper before submitting a final version for publication and evaluation. The two page limit also limits the effort required to revise these papers before final submission.

The final short paper is due one day after the final report is due, allowing time to incorporate any last minute report material into the short paper. During the short paper process it is necessary to remind the students that the materials they submit for the draft and final versions are different, and that the deadlines must be met. In this process, there have been few issues with post processing required. About $5-10 \%$ of groups have issues, usually submitting incorrect file types submitted: when asked, correct files are almost always sent quickly. A small mark is assigned based on the quality of the final short paper. The draft paper is not marked. The final paper is read by the instructor(s) and a (small) mark is assigned so that students take the exercise seriously.

\subsection{Templates and distribution}

Critical to the success of this publication exercise is to have all papers follow the same format and style. A paper template is provided to allow the students to focus on content rather than formatting. Importantly, the short paper template utilizes the type-setting tool LaTeX, rather than a more common word processing application. The template format is based on an IEEE two column conference paper format. Each student group authours their short paper using LaTeX and submits a set of LaTeX source files that contain their final short paper. These files are then used to generate a proceeding in a process that takes only a few hours.

The use of LaTeX greatly improves the proceedings compilation. The first year short papers were introduced in the course, the students were asked to produce them using a Word template. (The final reports were also submitted in MS Word (.doc) format.) It proved extremely time consuming and difficult to put together a professional proceeding based on a series of short papers saved as Word files. In fact, it was ultimately necessary to move the text body to another publishing application to produce the final proceeding. 
LaTeX was subsequently adopted as the course writing tool, and all course deliverables are now required to be authored using LaTeX. The goal is to encourage students to focus on the content and quality of their writing, rather than formatting or style, which can become significant preoccupations when using a common word processor like MS Word. The students are also introduced to a new open-source technical writing tool, and their initial unfamiliarity is meant to foster a better planned and more intentional writing process.

At the beginning of the course, students are given LaTeX instruction, tutorials and examples. During the course, the students start with a simple proposal, and move through written deliverables of increasing complexity. The ease of producing a professional, stylistically consistent proceeding from a collection of LaTeX papers is an added bonus. By the time they are writing their short papers at the end of the semester, they are very familiar with LaTeX and the level of support required is minor.

The templates used to publish the proceeding consist of:

- editor's template, where all group papers are linked by topic, pre and post matters are attached (preface, contents, etc.);

- author's template, which is a simplified subset of the editor's template allowing a group to edit and preview their two pages;

- package of styles for typesetting the body and references

The template used by the students is a simplified author's template which allows them to write and preview the contents of their submission. The relationship between the authour's template used by the students, and the more complete editor's template, is shown in figure 1. They create their content in a subdirectory of the template, named by group number.

Once their individual authoring is complete, the entire sub-directory containing all the source files is submitted online. Once all the LaTeX source files for the final short papers are submitted at deadline, they are unpacked into the editor's template, and compiled mostly as is. Generally, no additional editing is done on the content of the final short papers. The templates used by students assure that each paper conforms to the stylistic and length requirements. It takes approximately two to four hours to compile all the papers into a single professional proceeding, including the preface, table of contents and author index, and with individual titles, proper page numbering, figures, and references done automatically.

\section{Publication}

The completed proceedings is published online on the University of Guelph Library's Atrium archive (http://atrium.lib.uoguelph.ca), a publicly accessible long term electronic archive of university work. Library staff prepare unique links for each paper, and include meta data such as authour names and the key words from each paper. Each paper and the overall proceedings are provided with permanent URLs that are publicly accessible and therefore searchable using common search engines (e.g. Google, Yahoo).

\section{Impact}

Student perception of the two page paper is good. Many students appreciate the process of submitting a draft and having it reviewed without a mark being assigned. Written student comments on end-of-term surveys have noted that publishing the short paper encourages more care in the completion of the assignment and results in greater student engagement. Several students have also directly commented that they appreciate that there is a record of their work from the design course that can be shared with family, colleagues and potential employers.

The authours have received feedback from multiple students that they have included the short paper on their resumes or graduate school applications, and at that time they felt it was a valuable addition to their professional portfolio.

The proceedings is accessed and read by the public. The authours have been contacted many times by people who have read the papers and had questions about the student work. On two separate occasions, initial contacts based on short paper inquiries have led to course projects in future works.

\section{Conclusion}

Three years of experience publishing short papers that summarize student work in a design course suggest that this process helps students learn the importance of technical writing and develop their engineering communication skills. Including reviewing of a draft version of the paper helps students understand expectations and improve their paper before publication. The publication process itself has been a positive experience for both instructors and students. Knowing that the short paper will be published appears to provide an additional non-mark incentive for students to produce good work. The resulting publication is a valuable addition to student professional portfolios, while instructors and the 


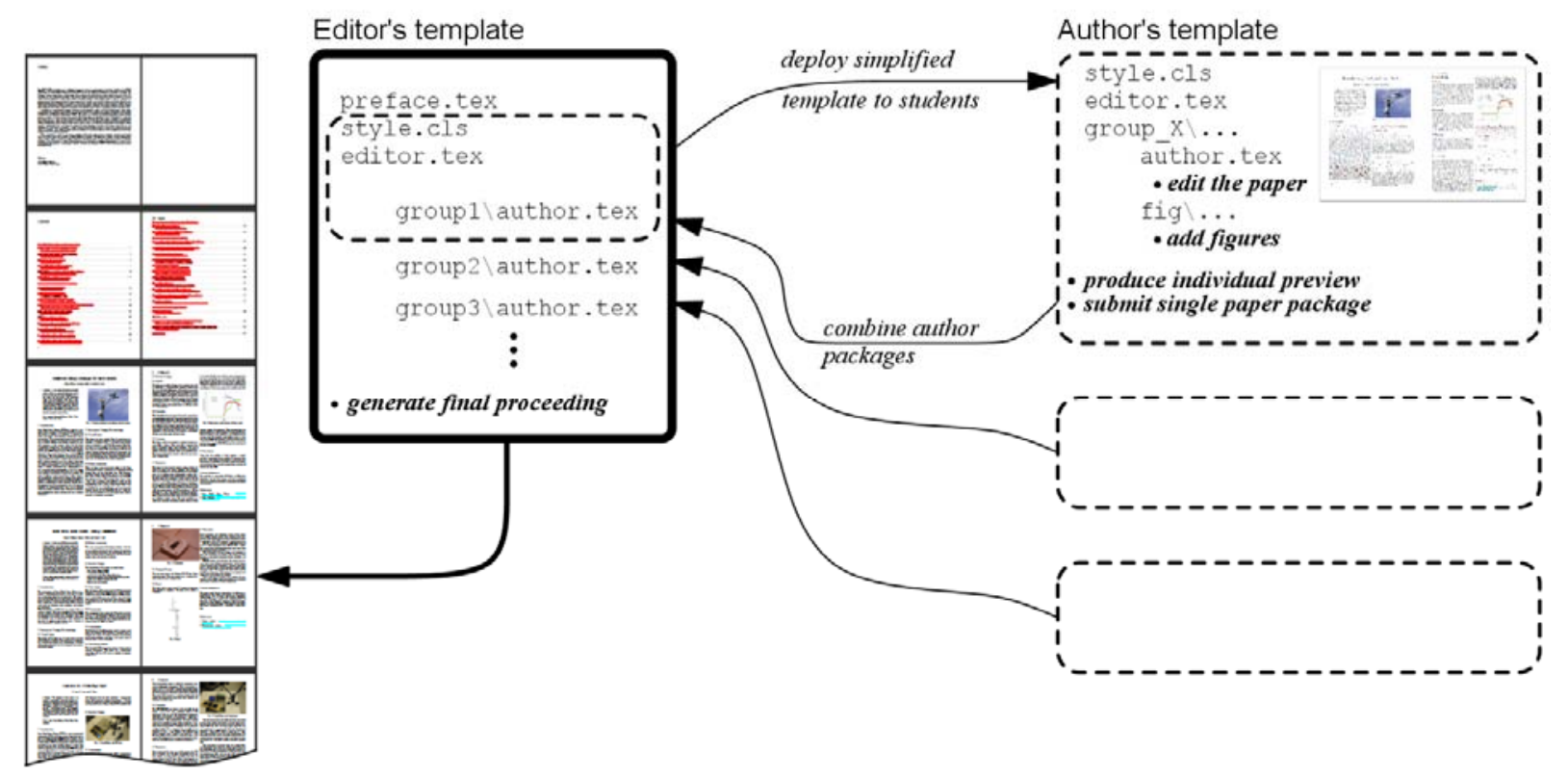

Figure 1. Process of combining short paper LaTeX templates into a single proceeding.

university benefit from the added publicity and availability of student work.

It is generally agreed that practicing engineers must have a well developed ability to communicate knowledge, ideas and processes. While some students already understand this, many engineering students do not. On a survey given at the end of the ENGG*3100 course, one student wrote

Engineers need to value communication and writing skills. We learned a lot about it in this course but I still feel like generally engineers here don't care about writing. How can you get students to care about this?

As this student noted, while initiatives like the short paper help teach a range of technical writing skills, it is still in the context of a single course. Mastery of technical writing requires practice, and significant improvement in the writing skills of graduating students will require integration of technical writing across the curriculum. 